\title{
Rational design of a highly efficient catalytic system for the production of 3'-phosphoadenosine-5'-phosphosulfate from ATP
}

\author{
Kaifang Liu ${ }^{1}$, Xiulai Chen ${ }^{1}$, Yunlu Zhong ${ }^{1}$, Jia Liu ${ }^{1}$, Guipeng $\mathrm{Hu}^{1}$, Cong $\mathrm{Gao}^{1}$, Liang \\ Guo $^{1}$, Wei Song ${ }^{1}$, and Liming Liu ${ }^{2}$ \\ ${ }^{1}$ Jiangnan University \\ ${ }^{2}$ Jiangnan Univ.
}

April 18, 2021

\begin{abstract}
The compound 3'-phosphoadenosine-5'-phosphosulfate (PAPS) serves as a sulfate group donor in the production of valuable sulfated compounds, such as glycosaminoglycan and oxamniquine. However, elevated costs and low conversion efficiency limit the industrial applicability of PAPS. Here, we designed and constructed an efficient and controllable catalytic system for the conversion of ATP (disodium salt) into PAPS without inhibition from by-products. In vitro and in vivo testing in Escherichia coli identified adenosine-5'-phosphosulfate kinase from Penicillium chrysogenum (PcAPSK) as the rate-limiting enzyme. Based on analysis of the catalytic steps and molecular dynamics simulations, a mechanism-guided "ADP expulsion" strategy was developed to generate an improved PcAPSK variant (L7), with a specific activity of $48.94 \mathrm{U} \cdot \mathrm{mg}$-1 and 73.27-fold higher catalytic efficiency $(\mathrm{kcat} / \mathrm{Km})$ that of the wild-type enzyme. The improvement was attained chiefly by reducing the ADP-binding affinity of PcAPSK, as well as by changing the enzyme's flexibility and lid structure to a more open conformation. By introducing PcAPSK L7 in an in vivo catalytic system, $73.59 \mathrm{mM}(37.32 \mathrm{~g} \cdot \mathrm{L}-1)$ PAPS was produced from $150 \mathrm{mM}$ ATP in $18.5 \mathrm{~h}$ using a 3-L bioreactor. The achieved titer is the highest reported to date and corresponds to a $98.13 \%$ conversion rate. The proposed strategy will facilitate industrial production of PAPS as well as the engineering of similar enzymes.
\end{abstract}

\section{Introduction}

The compound 3'-phosphoadenosine-5'-phosphosulfate (PAPS) acts as a sulfate group donor in the production of glucosinolate, heparin, chondroitin sulfate, and oxamniquine (Ji et al., 2020; Zhang, Lin, Huang, \& Linhardt, 2020). At present, PAPS can be produced either via metabolic or enzymatic synthesis. In the metabolic biosynthetic pathway (Harjes, Bayer, \& Scheidig, 2005; Sekulic, Dietrich, et al., 2007), ATP is converted to PAPS by ATP sulfurylase (ATPS) and adenosine-5'-phosphosulfate (APS) kinase (APSK). However, because ATP is an energy-rich compound with limited capacity to accumulate in cells, only $0.8-1.2$ $\mu$ mol PAPS is obtained per gram of cells (Badri et al., 2021; Badri, Williams, Xia, Linhardt, \& Koffas, 2019). Enzymatic synthesis (Burkart, Izumi, Chapman, Lin, \& Wong, 2000), which includes single-enzyme catalysis and dual-enzyme cascade catalysis, has been developed to further enhance PAPS output.

In single-enzyme catalysis, aryl sulfotransferase (ASST) converts 3'-phosphoadenosine-5'-phosphate (PAP) and $p$-nitrophenyl sulfate (PNPS) to PAPS (Jin et al., 2020; Z. Zhou et al., 2019). Over the past decade, various ASSTs from mammalian species and bacteria, such asStreptomyces sp. (Kaysser et al., 2010) and Escherichia coli (Malojcic, Owen, \& Glockshuber, 2014; Malojcic et al., 2008), have been identified and used to synthesize PAPS. However, ASST expression and affinity for PAP need to be improved (Berger, Guttman, Amar, Zarivach, \& Aharoni, 2011). To increase yields, fusion with the signal peptides Cex, YebF, and PelB has been applied, allowing for ASST secretion and fourfold higher expression (89.67 U.mL $\mathrm{mL}^{-1}$ ) (Z. Zhou et al., 2019). To improve affinity for PAP, random mutagenesis and molecular evolution of the PAP-binding pocket 
gate loop were applied based on the enzyme's crystal structure. PAP affinity and ASST catalytic efficiency were thus increased by 2.48 times and 12.50 times, respectively (Z. Zhou et al., 2019). Still, some hurdles remain, as the regeneration of PAPS requires coupling to sulfotransferases and prevents PAPS accumulation (An, Zhao, Wei, \& Zhou, 2017; Bao et al., 2015), while the elevated cost of PAP (38 $\$ \cdot \mathrm{mg}^{-1}$, Sigma) lowers its commercial appeal.

A relatively cheap dual-enzyme cascade catalysis using ATP as the substrate was developed. Given that the price of ATP is only 1/25 that of PAP, this strategy offers an appealing alternative (Datta et al., 2020). The catalysis process includes two steps: first, sulfate and ATP are converted by ATPS to adenosine-5'phosphosulfate (APS) and the by-product pyrophosphate (PPi) (Kang et al., 2018; Schmidt, 1977); second, APS kinase catalyzes the conversion of APS and ATP to PAPS and the by-product ADP (Badri et al., 2019). At present, this method has been used to synthesize PAPS at gram-level in the preparation of bioengineered heparin and chondroitin sulfate (Jian, Liu, Robert, \& reports, 2014; Jin et al., 2020). However, by-product inhibition and low enzymatic activity compel a substrate conversion rate of only $47 \%$ (An et al., 2017). To alleviate inhibition, by-product degradation and recycling have been attempted (Michael D. Burkart, 2000; X. Zhou, Chandarajoti, Pham, Liu, \& Liu, 2011). In one such example, the conversion rate was increased by $50 \%$, and $5.0 \mathrm{~g}^{*} \mathrm{~L}^{-1} \mathrm{PAPS}$ was generated when ADP was transformed back to ATP, using phosphoenolpyruvate (PEP) as a phosphate donor (An et al., 2017).

APSK is a key factor affecting catalytic efficiency of the dual-enzyme cascade and is characterized by a typical "lid structure" (Gay, Segel, \& Fisher, 2009). It catalyzes a sequential reaction, whereby ATP binds ahead of APS, and then PAPS leaves before ADP is released (Lansdon, Segel, \& Fisher, 2002). If ADP is not released in time, an APS-enzyme-ADP termination complex forms, resulting in a decrease in enzymatic activity (Lansdon et al., 2002; Mueller \& Shafqat, 2013). Protein crystallization (Ian J. MacRae, 2000; Poyraz et al., 2015), site-directed mutagenesis (Wang D. Z et al., 2016), and truncated mutagenesis (Ravilious, Westfall, \& Jez, 2013; Sekulic, Konrad, \& Lavie, 2007) have been used to study APSK. Secondary structure analysis of APSK from Arabidopsis thaliana showed that $\mathrm{Arg}^{93}$ was necessary for substrate recognition, and affinity for ADP was 217-times lower in the APSK ${ }^{\text {R93A }}$ mutant (Ravilious et al., 2013). Site-directed mutagenesis produced an Oryza sativa Os $\mathrm{APSK}^{\mathrm{C} 36 \mathrm{~A} / \mathrm{C} 69 \mathrm{~A}}$ mutant, whose $k$ cat was $43 \%$ lower than that in the wild-type, while the Km for APS was 1.6-fold higher (Wang D. Z et al., 2016). These studies focused mainly on APSK structure and its catalytic mechanism; however, attempts to improve catalytic performance of the enzyme have yielded only limited success.

In this study, a PAPS-producing catalytic system composed of a main module and an auxiliary module was designed and assessed in vivo. The main module converted ATP to PAPS, and the auxiliary module effectively eliminated by-product inhibition by hydrolyzing PPi to regenerate ATP from ADP. APSK was identified as the limiting step in this catalytic system due to delayed release of ADP. To further increase conversion efficiency, a mechanism-guided "ADP expulsion" strategy was developed to weaken the binding affinity of APSK for ADP and expand the bottleneck caused by its U-shaped release channel. Finally, by integrating the best variant in the cascade pathway, $73.6 \mathrm{mM}\left(37.3 \mathrm{~g}^{*} \mathrm{~L}^{-1}\right)$ PAPS was synthesized, using a 3-L fermenter, with $98.1 \%$ conversion.

\section{Materials and methods}

\section{Strains, plasmids, and media}

All strains and plasmids used in this study are listed in Table S1. The variants were constructed by whole plasmid PCR protocol with the main primers listed in Table S2 . The PCR system (100 $\mu$ l) was composed of template (100-150 ng), corresponding primers $(20 \mu \mathrm{M}$ with $2 \mu \mathrm{l})$, PrimeSTAR polymerase (1 $\mu \mathrm{l}$; Takara Biomedical Technology, Dalian, China), $5 \times$ PrimeSTAR Buffer $(20 \mu \mathrm{l})$, dNTP mix $(8 \mu \mathrm{l})$, and sterilized water. Next, DpnI was added to the PCR reaction mixture and incubated for $3 \mathrm{~h}$ at $37^{\circ} \mathrm{C}$ to eliminate the template plasmid. The digested product was transformed into Escherichia coli BL21(DE3) cells for the following screening or DNA sequencing (Genewiz, China). Luria-Bertani (LB) medium containing $5 \mathrm{~g} \cdot \mathrm{L}^{-1}$ yeast extract, $10 \mathrm{~g} \cdot \mathrm{L}^{-1}$ tryptone, and $10 \mathrm{~g} \cdot \mathrm{L}^{-1} \mathrm{NaCl}$ was used for strain selection and propagation. Terrific 
Broth (TB) medium containing $4 \mathrm{~g} \cdot \mathrm{L}^{-1}$ glycerin, $24 \mathrm{~g} \cdot \mathrm{L}^{-1}$ yeast extract, $12 \mathrm{~g} \cdot \mathrm{L}^{-1}$ tryptone, $2.31 \mathrm{~g} \cdot \mathrm{L}^{-1} \mathrm{KH}_{2} \mathrm{PO}_{4}$, and $16.43 \mathrm{~g} \cdot \mathrm{L}^{-1} \mathrm{~K}_{2} \mathrm{HPO}_{4}$ was used for protein expression. Chloramphenicol $\left(30 \mu \mathrm{g} \cdot \mathrm{mL}^{-1}\right)$, kanamycin $(50$ $\left.\mu \mathrm{g} \cdot \mathrm{mL}^{-1}\right)$, and ampicillin $\left(100 \mu \mathrm{g} \cdot \mathrm{mL}^{-1}\right)$, and IPTG $(0.4 \mathrm{mM})$ were added at the appropriate time.

\section{Homology modeling and molecular dynamics (MD) simulations}

The 3D structural of $P c$ APSK and its mutants were constructed based on its X-ray crystal structures (PDB ID: 1M7H) (Lansdon et al., 2002). The 3D structure of ADP was obtained from the ChemSpider Ultra 11.0 program (http://www.chemspider.com/ ). Nonpolar hydrogen atoms were added to the enzyme using the GROMACS 4.5.5 simulation package (http://www.gromacs.org/). The whole system was immersed in an explicit TIP 3P water box and extended with a thickness of at least $10 \mathrm{~A}$ from the dissolved atoms in each dimension. MD simulations were performed with GROMACS 4.5.5 and an AMBER03 force field following the three main steps of energy minimization, system equilibration, and production protocols. The $50 \mathrm{~ns} \mathrm{MD}$ simulations were performed using NAMD 2.12 (derived from "Not Another Molecular Dynamics program", by University of Illinois) with the Charmm27 force field with a 2 -fs time step at the temperature of $310 \mathrm{~K}$. The enzyme and product were assigned to separate energy groups, and the binding energies between them were calculated as total intergroup potential energy. The MD simulations results were analyzed in Visual Molecular Dynamics software (VMD 1.9.3, by University of Illinois). All images of the structures shown here were generated using PyMol and CAVER. The two flexible regions (A and B) identified by MD simulations were as follows: region A comprised residues from L33 to V43, and B region included residues from Y137 to H181.

\section{Analytical methods}

The optical density at $600 \mathrm{~nm}\left(\mathrm{OD}_{600}\right)$ was measured using a spectrophotometer. ATP, APS, and PAPS were quantified by high-performance liquid chromatography (HPLC). The analysis was performed with an HPLC system equipped with an polyamine column (YMC-Pack PolyamineII, $250 \mathrm{mmx} 4.6 \mathrm{~mm}$ ) and $0.7 \mathrm{mM}$ of $\mathrm{KH}_{2} \mathrm{PO}_{4}$ as the mobile phase. The flow rate of the mobile phase was $0.6 \mathrm{~mL} / \mathrm{min}$ and oven temperature was maintained at 30 throughout the operation with an UV detector at $254 \mathrm{~nm}$. Meanwhile, the product APS and PAPS were also analyzed by ESI-MS and the molecular weight was in accordance with that of standard.

More materials and methods are shown in Supporting Information.

\section{Results}

\section{Cascade design and in vitro construction of the PAPS biosynthesis pathway}

To efficiently produce PAPS, a sequential enzymatic cascade composed of a main module and an auxiliary module was designed (Figure 1A ). In the main module, ATP and sulfate were first converted to APS and PPi by ATPS. Thereafter, APS and ATP were converted to PAPS and ADP by APSK. In the auxiliary module, a pyrophosphatase (PPA) was introduced to hydrolyze PPi to Pi, and a polyphosphate kinase (PPK) was introduced to regenerate ATP from ADP; in this way, the inhibitory effect of the by-products (PPi and ADP) was eliminated. Using the BRENDA database, four different microorganisms were selected as the sources of each enzyme: ATPS from Kluyveromyces lactis (Kl ATPS), APSK fromPenicillium chrysogenum (Pc APSK), PPA from E. coli(Ec PPA), and PPK from Rhodobacter sphaeroides(Rs PPK). Following purification and in vitro evaluation, their optimal specific activity was determined (Table 1 andTable S3 ) .

To confirm the effectiveness of the main module in vitro , Kl ATPS and $P c$ APSK were mixed at a 1:1 molar ratio in the presence of $20 \mathrm{mM}$ ATP. After $10 \mathrm{~h}$, formation of $4.56 \mathrm{mM}$ PAPS as the final product was confirmed by nuclear magnetic resonance and mass spectrometry analyses (Figure $\mathbf{S} 1$ ), demonstrating the efficacy of using $K l$ ATPS and $P c$ APSK for converting ATP to PAPS. The effect of varying the $K l$ ATPS:Pc APSK ratio on PAPS titer was investigated with $K l$ ATPS activity fixed at $3.0 \mathrm{U}^{*} \mathrm{~mL}^{-1}$. The highest PAPS titer $(6.06 \mathrm{mM}$, conversion rate $59.6 \%)$ was achieved when $P c$ APSK activity was $4.5 \mathrm{U}^{*} \mathrm{~mL}^{-1}$ (Figure 1B ). This result indicated an optimal in vitro $K l$ ATPS:Pc APSK ratio of 1:1.5. Next, the effect of adding $E c$ 
PPA at 2.5, 3.0, 3.5, 4.0, 4.5, and 5.0 $\mathrm{U}^{*} \mathrm{~mL}^{-1}$ on PAPS titer was examined (Figure 1C ). Addition of 4.0 $\mathrm{U}^{*} \mathrm{~mL}^{-1}$ Ec PPA to the main module led to a PAPS titer of $7.18 \mathrm{mM}$ from $20 \mathrm{mM}$ ATP, with conversion rate of $81.8 \%$. Furthermore, when $7.0 \mathrm{U}^{*} \mathrm{~mL}^{-1}$ Rs PPK was added to the in vitroconversion broth, the PAPS titer increased to $8.05 \mathrm{mM}$, with a conversion rate of $92.5 \%$ (Figure 1D ). Taken together, the optimal $\mathrm{Kl}$ ATPS:Pc APSK:Ec PPA:Rs PPK ratio was approximately 3:4.5:4:7.

\section{In vivo construction of the PAPS biosynthesis pathway}

To prove the effectiveness of the proposed catalytic pathway in vivo, the genes encoding $K l$ ATPS and $P c$ APSK in the main enzyme module and Ec PPA and $R s$ PPK in the auxiliary module were inserted into pACYCDuet-1 and pETDuet-1 plasmids, respectively. These were then used to transform E. coli BL21 (DE3), resulting in E. coli strain 01. Co-expression of the four enzymes was confirmed by protein gel electrophoresis of cell-free lysates. Then, the conversion performance of strain E. coli 01 was investigated with $30 \mathrm{~g}^{*} \mathrm{~L}^{-1}$ wet cell (treated with $1 \mathrm{~g}^{*} \mathrm{~L}^{-1}$ Tween 80 ) at $30 \mathrm{degC}$, it was found that the PAPS titer increased from 6.03 to $11.59 \mathrm{mM}$ as the substrate ATP concentration was increased from 20 to $60 \mathrm{mM}$ (Figure 2A ). When the ATP concentration was increased $>60 \mathrm{mM}$, the PAPS titer did not augment any further. In contrast, the conversion rate of PAPS decreased from $60.13 \%$ to $25.62 \%$, when the ATP concentration increased from 20 to $80 \mathrm{mM}$. This drop was due to APS, an intermediate of the enzymatic cascade, which accumulated at increasing amounts (from 3.86 to $16.07 \mathrm{mM}$ ) in the conversion broth (Figure 2A ). Intracellular enzymatic activity of $K l$ ATPS, Pc APSK, Ec PPA, and $R s$ PPK in E. coli 01 was 135.06, 12.75, 188.19, and 243.31 $\mathrm{U}^{*} \mathrm{~g}^{-1}$ wet cells, respectively (the ratio was 3:0.28:4.18:5.41) (Figure 2B ). This finding revealed the need to increase the intracellular enzymatic activity of $R s$ PPK and $P c$ APSK.

Previous studies had shown that $R s$ PPK could easily form inclusion bodies in cells. Therefore, four molecular chaperones (SUMO, MBP, TrxA, and GST) involved in expression of soluble proteins were co-expressed with $R s$ PPK to increase its activity in the cytoplasm. TrxA alone caused the intracellular activity of $R s$ PPK to increase from 243.31 (E. coli 01 ) to $317.38 \mathrm{U}^{*} \mathrm{mg}^{-1}$ wet cells (E. coli 04) (Figure 2C), while $\mathrm{Kl}$ ATPS, $P c$ APSK, and $E c$ PPA reached 132.61, 12.85, and $186.63 \mathrm{U}^{*} \mathrm{~g}^{-1}$ wet cells, respectively (corresponding to a 3:0.29:4.22:7.18 ratio) (Figure 2A ). To improve Pc APSK intracellular activity, its expression was increased by adding six ribosome-binding site (RBS) sequences (RBS1-RBS6) of different intensities (Table S4 ). RBS5 produced the best results and increased Pc APSK activity from 10.75 (E. coli 01) to 22.47 $\mathrm{U}^{*} \mathrm{~g}^{-1}$ wet cells (E. coli 06) (Figure 2D ). However, increasing the expression of Pc APSK decreased the transformation efficiency of the system (Figure 2D ). Moreover, the intracellular activity of the other three enzymes to drop significantly to 95.07 ( $K l$ ATPS), 87.35 (Ec PPA), and 194.05 (Rs PPK) U*g ${ }^{-1}$ wet cells (Figure 2A ). These results suggested that increasing enzyme expression alone had limited efficacy on improving overall performance and might lead to an imbalance in intracellular resource allocation. To overcome this limitation, it is necessary to improve the inherent properties of $P c$ APSK through protein engineering.

\section{ADP expulsion increases PcAPSK activity}

To increase $P c$ APSK activity, the enzyme's catalytic mechanism and structural characteristics were first studied. The catalytic process of $P c$ APSK can be divided into three steps (Figure 3A ): (i) ATP binds to the enzyme, causing the lid structure to close; (ii) APS reaches the binding site and reacts with ATP; and (iii) the lid structure is opened to first release PAPS and then ADP. If ADP is not released in time, a complex (Enzyme-ADP-APS) will be formed and hinders the catalysis progress. To study the interaction between $P c$ APSK and ADP, ADP was docked into the $P c$ APSK based on its crystal structure (PDB ID: 1M7H) (Figure 3B ), and a bottleneck of $6.4 \mathrm{~A}$ in the release channel was identified using CAVER (Figure 3C). Then, molecular dynamics (MD) simulation was performed to calculate the root mean square fluctuation (RMSF) values and conformation of $P c$ APSK. As shown in Figure 3D, two regions (A and B) displayed higher RMSF values, suggesting that these two motifs could undergo noticeable movement and influence protein conformation. In addition, MD simulations revealed a conformational change of the lid, which regulated the release of ADP by controlling the "closed-open" movement (Figure 3E ). ADP was immobilized by residues, whose binding free energy reached $-55.81 \mathrm{kcal}^{*} \mathrm{~mol}^{-1}$ in the closed conformation. 
These results proved that the release of ADP was unfavorable; hence, promoting it might speed up the catalytic reaction.

Based on the structure of ADP-APSK and MD simulations, an "ADP expulsion" strategy was designed to promote the release of ADP by (i) reducing the binding affinity of target residues, (ii) changing the conformation of the lid to widen the release tunnel. Thirty-four candidate residues in regions A and B were selected for alanine scanning (Figure 4A ). Six residues whose activity was [?]30\% higher were identified: binding residues S36A (35.2\%), K38A (74.7\%), and T40A135.17 (57.2\%) in region A, and lid constituent residues K151A (107.2\%), D139A (43.6\%), and G167A (67.3\%) in region B. Then, two smart mutation libraries (A and B) were constructed and screened for NNK-based site-saturation mutagenesis (Figure 4B ). In library A, saturation mutation of K38 increased APSK activity by 5.31 -fold in variant $\mathrm{L} 1_{\mathrm{K} 38 \mathrm{G}}$, whereas iterative saturation mutation of K38 and T40 produced mutant L2 $2_{\mathrm{K} 38 \mathrm{G} / \mathrm{T} 40 \mathrm{~S}}$, which presented 12.12-fold higher activity. Notably, when mutation S36 was introduced into L2, resulting in variant $\mathrm{L} 3_{\mathrm{S} 36 \mathrm{G} / \mathrm{K} 38 \mathrm{G} / \mathrm{T} 40 \mathrm{~S}}$, a significant drop in activity, amounting to only 4.99-fold of the wild-type (L0), was observed (Figure 4B ). In library B, saturation mutation of K151 increased the activity by 4.50 -fold in variant $\mathrm{L}_{\mathrm{K} 151 \mathrm{~V}}$, and saturation mutation of G167 increased it by 7.80-fold in variant L5 $5_{\mathrm{K} 151 \mathrm{~V} / \mathrm{G} 167 \mathrm{I}}$. Iterative saturation mutation of K151, G167, and D139 produced mutant L6 1 D139V/K151V/G167I, which presented 19.65-fold higher APSK activity. Finally, the

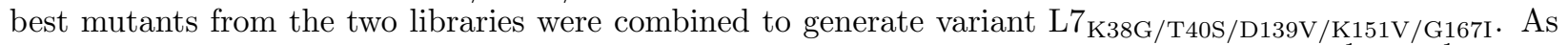
shown in Table 2 , variant L7 exhibited a 31.89-fold increase in $k c a t$, from 0.61 to $19.45 \mathrm{~s}^{-1 *} \mathrm{mM}^{-1}$, and a 45.74-fold higher specific activity $\left(48.94 \mathrm{U}^{*} \mathrm{mg}^{-1}\right)$ compared to L0.

To explain the improved $P c$ APSK activity from a mechanism point of view, binding affinity and release channel width were compared between variants L0 and L7. In the latter, binding affinity for ADP was reduced due to an increase in binding energy from -55.82 to $-21.57 \mathrm{kcal}^{*} \mathrm{~mol}^{-1}$, as calculated by MD simulations. As shown in Figure 4C, the average number of hydrogen bonds decreased from 31.1 to 27.3, which might have led to a weaker $\mathrm{ADP}$-enzyme interaction. As a result, the root mean square distance increased from 0.55 to 0.63 A (Figure 4D), suggesting a reduced stability of $P c$ APSK. Analysis of the release channels revealed that the bottleneck in L7 increased by nearly $0.73 \mathrm{~A}$ to $7.13 \mathrm{~A}$ (Figure $4 \mathbf{E}$ ) and was more conducive to the release of ADP. In addition, the lid of the L7 variant changed to a more open state following engineering of the lid and hinge residues (Figure $\mathbf{4 F}$ ). These, caused a kink in the loop (from I162 to A170) that shifted two $\alpha$-helix moieties to a more open state $(0.52$ and $0.55 \AA$, respectively). In particular, compared to the L0 variant, L7 showed an increase in RMSF of nearly 0.18 and $0.25 \AA$ around regions A and B, respectively (Figure 4G ). These results indicate that the change in hinge residues led to a more flexible channel, which favored ADP release. In summary, the mechanism promoting the release of ADP might have resulted from a lower binding affinity due to weaker inner interactions, as well as increased flexibility of hinge residues, leading to a more open conformation of the lid and a wider release channel.

\section{One-pot production of PAPS at a 3-L scale}

To demonstrate the in vivo applicability of the proposed strategy, the L7 variant was introduced in a wholecell catalytic system, resulting in strain $E$. coli 11 . Finally, the intracellular activity of $K l$ ATPS, Pc APSK, Ec PPA and Rs PPK could be controlled to 166.32, 253.08, 225.91, and $403.26 \mathrm{U}^{*} \mathrm{~g}^{-1}$ wet cells, respectively (Figure 5A ). Optimal operational conditions were determined as $\mathrm{pH} 8.0$ and $35 \mathrm{deg}$ C. As shown in Figure S2, increasing substrate concentration from 100 to $150 \mathrm{mM}$ achieved a conversion rate $>97 \%$, but a further increase to $200 \mathrm{mM}$ caused the conversion rate to drop to $80.3 \%$. Therefore, $150 \mathrm{mM}$ substrate was considered optimal for conversion. The strainsE. coli 04 (L0) and 11 (L7) were each grown in a 3-L bioreactor under optimal conditions, with air flow of $4.5 \mathrm{~L}^{*} \mathrm{~min}^{-1}$ and shaking speed of $210 \mathrm{r}^{*} \mathrm{~min}^{-1}$ (Figure 5B ). Escherichia coli 11 produced $73.59 \mathrm{mM}\left(37.32 \mathrm{~g}^{*} \mathrm{~L}^{-1}\right)$ PAPS after incubation for $18.5 \mathrm{~h}$, corresponding to a $98.1 \%$ conversion rate and space-time yield of $1.8 \mathrm{~g}^{*} \mathrm{~L}^{-1 *} \mathrm{~h}^{-1}$. In comparison, E. coli 04 could produce only 37.94 $\mathrm{mM}\left(19.25 \mathrm{~g}^{*} \mathrm{~L}^{-1}\right)$ PAPS after $20.5 \mathrm{~h}$, corresponding to a $50.6 \%$ conversion efficiency and a space-time yield of $0.94 \mathrm{~g}^{*} \mathrm{~L}^{-1 *} \mathrm{~h}^{-1}$. In addition, almost no APS could be detected in the reaction solution of $E$. coli 11 , whereas $22.35 \mathrm{mM}$ APS was found in the reaction solution of $E$. coli 04 . These results showed that protein engineering of $P c$ APSK in the dual-enzyme catalytic cascade had successfully eliminated the rate-limiting 
step in PAPS production.

\section{Discussion}

In this study, a new dual-enzyme catalytic system for in vivosynthesis of PAPS was developed that comprised a main enzyme module for the conversion of ATP into PAPS and an auxiliary module for the removal of by-product inhibition. Pc APSK was identified as the rate-limiting enzyme in this cascade due to the delayed release of ADP. To overcome this limitation and improve catalytic efficiency, a rational "ADP expulsion" strategy was applied. Accordingly, the ADP-binding affinity was weakened, and the binding channel was expanded to promote the release of ADP, resulting in a 45.74-fold higher activity in the APSK L7 variant. The latter was introduced in an E. colicatalytic system, whereby it successfully converted ATP to PAPS. This study describes not only a feasible method for the industrial production of PAPS but also a valuable strategy for engineering similar enzymes.

A synthetic and controllable catalytic system was designed for the synthesis of PAPS. The proposed catalytic system eliminates pathway inhibition from by-products by converting these by-products into substrates for reuse. During the conversion of ATP to PAPS, two by-products, PPi and ADP, inhibit the performance of ATPS and APSK (Bao et al., 2015). To eliminate such by-product inhibition, PPA and Nudix hydrolase have been used as PPi and ADP hydrolases, respectively (Hong et al., 2014; W. Xu, Dunn, O, Handley, Smith, \& Bessman, 2006). Previously, a complex ATP conversion system was constructed using 3-bromopyruvic acid as a cheap substrate for PEP-K ${ }^{+}$, which then acted as a phosphate donor for ADP (An et al., 2017). In this study, an auxiliary module comprising PPA and PPK was designed. PPA hydrolyzed PPi to phosphate, and PPK phosphorylated ADP to ATP through a one-step reaction that employed low-cost short-chain polyphosphate PolyP(6) as phosphate donor. In contrast to other methods for producing PAPs via the onepot process, the present catalytic system is composed of two independent modules, whose strength can be adjusted by controlling their intracellular expression to balance the production process.

An effective protein engineering strategy was developed to improve $P c$ APSK activity. To date, protein crystallization (Ian J. MacRae, 2000; Poyraz et al., 2015), site directed mutagenesis Wang D. Z et al., 2016), and truncated mutagenesis (Ravilious et al., 2013; Sekulic, Konrad, et al., 2007) have been used to characterize APSK. However, these studies focused mainly on the structure and catalytic mechanism of APSK, and no effective strategies for improving its catalytic performance have been proposed. Here, a mechanism-guided "ADP expulsion" strategy was developed by combining information about conformational dynamics and crystal structure of the protein (Ian J. MacRae, 2000; Lansdon et al., 2002). The strategy included three steps: (i) computer- and protein structure-assisted binding energy calculation and release channel identification; (ii) identification of six key mutation hotspots affecting binding energy and release channels; and (iii) construction of two mutation libraries aimed at weakening the binding energy through looser inner interactions and an expanded release channel. As a result, the specific activity and $k c a t / K m$ of the optimal L7 variant were 46.39-fold and 73.27-fold higher than those in wild-type Pc APSK, respectively. This approach is in line with a recent trend in protein engineering that focuses on construction of small and smart libraries while reducing the size of the mutation library and improving evolution efficiency $(\mathrm{Li}$, $\mathrm{Qu}$, Sun, \& Reetz, 2019; Sun, Lonsdale, Ilie, Li, \& Reetz, 2016). Compared to traditional mutagenesis, this method is more rational as it relies on tunnel identification (Song et al., 2020; Yuan et al., 2019), conformational dynamics (Yang et al., 2017), specific hotspot scanning (Xu , Cen, Singh, Fan, \& Wu, 2019; J. Xu et al., 2019), and saturation mutagenesis. Overall, this protein engineering strategy could greatly improve the performance of enzymes with a release channel or lid structure.

The study provides a simple and efficient method for in vivo ATP conversion to PAPS. Efficient PAPS production has relied on the use of either PAP or ATP as the substrate (Kang et al., 2018). In the first case, PAP and PNPS are converted to PAPS by ASST (T. Wang, Liu, \& Voglmeir, 2020); however, this method does not allow for large-scale PAPS production because of the high cost of PAP and the coupling with sulfotransferases (Xiong et al., 2013). ATP is a much cheaper substrate and, therefore, has been applied in the present study together with ATPS, APSK, PPA, and pyruvate kinase II as catalysts. The phospho-donor PEP-K ${ }^{+}$was generated by organic synthesis. Using $0.05 \mathrm{mg}^{*} \mathrm{~mL}^{-1}$ of each purified enzyme and PEP-K ${ }^{+}, 5$ 
$\mathrm{g}^{*} \mathrm{~L}^{-1}$ of PAPS was generated in $6 \mathrm{~h}$ with a productivity of $0.83 \mathrm{~g}^{*} \mathrm{~L}^{-1 *} \mathrm{~h}^{-1}$ (An et al., 2017). In this study, the main module and auxiliary module were assembled and transformed into an E. coli strain, the rate-limiting enzyme and catalytic conditions were optimized, and a stable whole-cell PAPS catalytic synthesis system was constructed. Finally, $73.59 \mathrm{mM}\left(37.32 \mathrm{~g}^{*} \mathrm{~L}^{-1}\right)$ PAPS was produced in $18.5 \mathrm{~h}$ with a conversion rate of $98.1 \%$ and productivity of $1.75 \mathrm{~g}^{*} \mathrm{~L}^{-1 *} \mathrm{~h}^{-1}$ at a 3 -L scale. Therefore, the biocatalytic process used in this study provides an attractive strategy for the transformation of ATP into high-value PAPS at a fraction $(1 / 5000)$ of the cost of commercial sources and thus may remarkably facilitate the industrial production of PAPS.

\section{Acknowledgments}

This work was financially supported by the Youth Program of National Natural Science Foundation of China (21808083), the Science Fund for Creative Research Groups of the National Natural Science Foundation of China (32021005), the National Key R \& D Program of China (2018YFA0901400), the National Key R \& D Program of China (2020YFA0908500).

\section{References}

An, C., Zhao, L., Wei, Z., \& Zhou, X. (2017). Chemoenzymatic synthesis of 3 '-phosphoadenosine-5 'phosphosulfate coupling with an ATP regeneration system. Applied Microbiology and Biotechnology, 101 (20), 7535-7544. doi :10.1007/s00253-017-8511-2

Badri, A., Williams, A., Awofiranye, A., Datta, P., Xia, K., He, W., . . . Koffas, M. A. G. (2021). Complete biosynthesis of a sulfated chondroitin in Escherichia coli . Nature Communications, 12 (1), 1389-1389. doi :10.1038/s41467-021-21692-5

Badri, A., Williams, A., Xia, K., Linhardt, R. J., \& Koffas, MAG. (2019). Increased 3'-phosphoadenosine-5'phosphosulfate levels in engineered Escherichia coli cell lysate facilitate the in vitro synthesis of chondroitin sulfate A. Biotechnology Journal, 14 (9), 111-119. doi :10.1002/biot.201800436

Bao, F., Yan, H., Sun, H., Yang, P., Liu, G., \& Zhou, X. (2015). Hydrolysis of by-product adenosine diphosphate from 3'-phosphoadenosine-5'-phosphosulfate preparation using Nudix hydrolase NudJ. Appl Microbiol Biotechnol, 99 (24), 10771-10778.doi :10.1007/s00253-015-6911-8

Berger, I., Guttman, C., Amar, D., Zarivach, R., \& Aharoni, A. (2011). The molecular basis for the broad substrate specificity of human sulfotransferase 1A1. Plos One, 6 (11), 1-10.doi :10.1371/journal.pone.0026794

Burkart, M. D., Izumi, M., Chapman, E., Lin, C. H., \& Wong, C. H. (2000). Regeneration of PAPS for the enzymatic synthesis of sulfated oligosaccharides. Journal of Organic Chemistry, 65 (18), 5565-5574. doi $: 10.1021 /$ jo000266o

Datta, P., Fu, L., He, W., Koffas, M. A. G., Dordick, J. S., \& Linhardt, R. J. (2020). Expression of enzymes for 3 '-phosphoadenosine-5 '-phosphosulfate (PAPS) biosynthesis and their preparation for PAPS synthesis and regeneration. Applied Microbiology and Biotechnology, 104 (16), 7067-7078.doi :10.1007/s00253-02010709-6

Gay, S. C., Segel, I. H., \& Fisher, A. J. (2009). Structure of the two-domain hexameric APS kinase from Thiobacillus denitrificans : structural basis for the absence of ATP sulfurylase activity. Acta Crystallographica Section D-Structural Biological Crystallography, 65 (10), 1021-1031. doi :10.1107/s0907444909026547

Harjes, S., Bayer, P., \& Scheidig, A. J. (2005). The crystal structure of human PAPS synthetase 1 reveals asymmetry in substrate binding. Journal of Molecular Biology, 347 (3), 623-635.doi :10.1016/j.jmb.2005.01.005

Hong, M. K., Ribeiro, A., Kim, J. K., Ngo, H., Kim, J., Lee, C. H., . . . Ramos, M. J. (2014). Divalent metal ion-based catalytic mechanism of the Nudix hydrolase Orf153 (YmfB) from Escherichia 
coli .Acta Crystallographica Section D-Structural Biological Crystallography, 70 (5), 1297-1310. doi :10.1107/s1399004714002570

Ian J. MacRae, I. H. S., and Andrew J. Fisher. (2000). Crystal structure of adenosine 5'-phosphosulfate kinase from Penicillium chrysogenum . Biochemistry, 39 , 1613-1621. dio : 10.1021/bi9924157

Ji, Y., Zhang, S., Qiao, M., Jiao, R., Li, J., Song, P., \& Huang, H. (2020). Synthesis of structurally defined chondroitin sulfate: paving the way to the structure-activity relationship studies.Carbohydrate Polymers, 248 (15), 1-11.doi :10.1016/j.carbpol.2020.116796

Jian, Liu, Robert, J., \& reports, L. J. N. p. (2014). Chemoenzymatic synthesis of heparan sulfate and heparin. Natural Product Reports, 31 (12), 1676-1685. doi : 10.1002/chin.201504262

Jin, X., Li, Q., Wang, Y., Zhang, W., Xu, R., Li, J., . . . Kang, Z. (2020). Optimizing the sulfationmodification system for scale preparation of chondroitin sulfate A. Carbohydrate Polymers, 246 (18), 116570. doi $: 10.1016 / \mathrm{j}$. carbpol.2020.116570

Kang, Z., Zhou, Z., Wang, Y., Huang, H., Du, G., \& Chen, J. (2018). Bio-based strategies for producing glycosaminoglycans and their oligosaccharides. Trends in Biotechnology, 36 (8), 806-818.doi $: 10.1016 /$ j.tibtech.2018.03.010

Kaysser, L., Eitel, K., Tanino, T., Siebenberg, S., Matsuda, A., Ichikawa, S., \& Gust, B. (2010). A new arylsulfate sulfotransferase involved in liponucleoside antibiotic biosynthesis inStreptomycetes . Journal of Biological Chemistry, 285 (17), 12684-12694. doi :10.1074/jbc.M109.094490

Lansdon, E. B., Segel, I. H., \& Fisher, A. J. (2002). Ligand-induced structural changes in adenosine 5'-phosphosulfate kinase fromPenicillium chrysogenum . Biochemistry, 41 (46), 13672-13680. doi :10.1021/bi026556b

Li, A., Qu, G., Sun, Z., \& Reetz, M. T. J. A. C. (2019). Statistical analysis of the benefits of focused saturation mutagenesis in directed evolution based on reduced amino acid alphabets. Acs Catalysis . 2019, 9 (9), 7769-7778. doi :10.1021/acscatal.9b02548

Malojcic, G., Owen, R. L., \& Glockshuber, R. (2014). Structural and mechanistic insights into the PAPSindependent sulfotransfer catalyzed by bacterial aryl sulfotransferase and the role of the DsbL/Dsbl system in its folding. Biochemistry, 53 (11), 1870-1877.doi :10.1021/bi401725j

Malojcic, G., Owen, R. L., Grimshaw, J. P. A., Brozzo, M. S., Dreher-Teo, H., \& Glockshuber, R. (2008). A structural and biochemical basis for PAPS-independent sulfuryl transfer by aryl sulfotransferase from uropathogenic Escherichia coli . Proceedings of the National Academy of Sciences of the United States of America, 105 (49), 19217-19222. doi :10.1073/pnas.0806997105

Michael D. Burkart, M. I., Eli Chapman, Chun-Hung Lin, \& Chi-Huey Wong. (2000). Regeneration of PAPS for the enzymatic synthesis of sulfated. The Journal of Organic Chemistry, 65 (18): 5565-5574. dio : 10.1021/jo000266o

Mueller, J. W., \& Shafqat, N. (2013). Adenosine-5'-phosphosulfate-a multifaceted modulator of bifunctional 3'-phospho-adenosine-5'-phosphosulfate synthases and related enzymes. The FEBS Journal, 280 (13), 30503057.doi : $10.1111 /$ febs. 12252

Poyraz, O., Brunner, K., Lohkamp, B., Axelsson, H., Hammarstrom, L. G., Schnell, R., \& Schneider, G. (2015). Crystal structures of the kinase domain of the sulfate-activating complex in Mycobacterium tuberculosis . Plos One, 10 (3),1-19.doi :10.1371/journal.pone.0121494

Ravilious, G. E., Westfall, C. S., \& Jez, J. M. (2013). Redox-linked gating of nucleotide binding by the Nterminal domain of adenosine 5'-phosphosulfate kinase. Journal of Biological Chemistry, 288 (9), 6107-6115. doi $: 10.1074 /$ jbc.M112.439414 
Schmidt, A. (1977). Assimilatory sulfate reduction via 3'-phosphoadenosine-5'-phosphosulfate (PAPS) and adenosine-5'-phosphosulfate (APS) in blue-green algae. FEMS Microbiology Letters, 1 (3), 137-140.doi :10.1111/j.1574-6968.1977.tb00599.x

Sekulic, N., Dietrich, K., Paarmann, I., Ort, S., Konrad, M., \& Lavie, A. (2007). Elucidation of the active conformation of the APS-kinase domain of human PAPS synthetase 1. Journal of Molecular Biology, 367 (2), 488-500. doi :10.1016/j.jmb.2007.01.025

Sekulic, N., Konrad, M., \& Lavie, A. (2007). Structural mechanism for substrate inhibition of the adenosine 5'-phosphosulfate kinase domain of human 3'-phosphoadenosine 5'-phosphosulfate synthetase 1 and its ramifications for enzyme regulation. Journal of Biological Chemistry, 282 (30), 22112-22121. doi :10.1074/jbc.M701713200

Song, W., Xu, X., Gao, C., Zhang, Y., Wu, J., Liu, J., . . . Liu, L. (2020). Open gate of Corynebacterium glutamicum threonine deaminase for efficient synthesis of bulky alpha-keto acids. Acs Catalysis, 10 (17), 9994-10004. doi :10.1021/acscatal.0c01672

Sun, Z., Lonsdale, R., Ilie, A., Li, G., \& Reetz, M. T. J. A. C. (2016). Catalytic asymmetric reduction of difficult-to-reduce ketones: triple code saturation mutagenesis of an alcohol dehydrogenase. Acs Catalysis, 6 (3), 1598-1605. doi : 10.1021/acscatal.5b02752

Wang D. Z, Chen G. G, Lu L. J, Jiang Z. J, Rao Y. C, Sun M. H. (2016). In vitro functional study of rice adenosine 5'-phosphosulfate kinase.Rice Science, 23 (3), 152-159. doi:10.1016/j.rsci.2016.04.002

Wang, T., Liu, L., \& Voglmeir, J. (2020). Chemoenzymatic synthesis of ultralow and lowmolecular weight heparins. Biochimica Et Biophysica Acta-Proteins and Proteomics, 1868 (2), 1-11.doi :10.1016/j.bbapap.2019.140301

Xiong, J., Bhaskar, U., Li, G., Fu, L., Li, L., Zhang, F., . . . Linhardt, R. J. (2013). Immobilized enzymes to convert $\mathrm{N}$-sulfo, $\mathrm{N}$-acetyl heparosan to a critical intermediate in the production of bioengineered heparin. Journal of Biotechnology, 167 (3), 241-247.doi :10.1016/j.jbiotec.2013.06.018

Xu , J., Cen, Y., Singh, W., Fan, J., \& Wu, Q. (2019). Stereodivergent protein engineering of a lipase to access all possible stereoisomers of chiral esters with two stereocenters. Journal of the American Chemical Society, 141 (19), 7934-7945. doi :10.1021/jacs.9b02709

Xu, J., Peng, Y., Wang, Z., Hu, Y., Fan, J., Zheng, H., Wu, Q. (2019). Exploiting cofactor versatility to convert a FAD-dependent baeyer-villiger monooxygenase into a ketoreductase. Angewandte Chemie International Edition, 58 (41), 14499-14503.doi :10.1002/anie.201907606

Xu, W., Dunn, C. A., O, Handley, S. F., Smith, D. L., \& Bessman, M. J. (2006). Three new nudix hydrolases from Escherichia coli . Journal of Biological Chemistry, 281 (32), 22794-22798.doi :10.1074/jbc.M603407200

Yang, B., Wang, H., Song, W., Chen, X., Liu, J., Luo, Q., \& Liu, L. (2017). Engineering of the conformational dynamics of lipase to increase enantioselectivity. Acs Catalysis, 7 (11), 7593-7599.doi :10.1021/acscatal.7b02404

Yuan, Y., Song, W., Liu, J., Chen, X., Luo, Q., \& Liu, L. (2019). Production of $\alpha$-ketoisocaproate and $\alpha$-keto- $\beta$-methylvalerate by engineered L-amino acid deaminase. ChemCatChem, 11 (10), 2464-2472. doi $: 10.1002 /$ cctc. 201900259

Zhang, X., Lin, L., Huang, H., \& Linhardt, R. J. (2020). Chemoenzymatic synthesis of glycosaminoglycans. Accounts of Chemical Research, 53 (2), 335-346. doi :10.1021/acs.accounts.9b00420

Zhou, X., Chandarajoti, K., Pham, T. Q., Liu, R., \& Liu, J. (2011). Expression of heparan sulfate sulfotransferases in Kluyveromyces lactis and preparation of 3'-phosphoadenosine-5'-phosphosulfate. Glycobiology, 21 (6), 771-780. doi :10.1093/glycob/cwr001 
Zhou, Z., Li, Q., Xu, R., Wang, B., Du, G., \& Kang, Z. (2019). Secretory expression of the rat aryl sulfotransferases IV with improved catalytic efficiency by molecular engineering. Biotech, 9 (6), 111-117. doi :10.1007/s13205-019-1781-x

Tables

Table 1 Kinetic constants of $K l$ ATPS, $P c$ APSK,Ec PPA and $R s$ PPK

\begin{tabular}{lllll}
\hline Parameters & $K m(\mathrm{mM})$ & $k c a t\left(\mathrm{~s}^{-1}\right)$ & $k c a t / K m\left(\mathrm{~s}^{-1} \cdot \mathrm{mM}^{-1}\right)$ & $\begin{array}{l}\text { Specific activity } \\
\left(\mathrm{U} \cdot \mathrm{mg}^{-1} \text { protein }\right)\end{array}$ \\
\hline KlATPS & 0.62 & 6.85 & 11.05 & 10.73 \\
PcAPSK & 1.35 & 0.61 & 0.45 & 1.07 \\
EcPPA & 0.32 & 27.06 & 84.56 & 50.47 \\
RsPPK & 0.41 & 38.42 & 93.71 & 63.85 \\
\hline
\end{tabular}

Table 2 Results of site-directed mutagenesis experiments

\begin{tabular}{|c|c|c|c|c|c|c|}
\hline Mutant & Mutant & mutation & $K m(\mathrm{mM})$ & Kcat $\left(\mathrm{s}^{-1}\right)$ & $\begin{array}{l}k c a t / K m \\
\left(\mathrm{~s}^{-1} \cdot \mathrm{mM}^{-1}\right)\end{array}$ & $\begin{array}{l}\text { Specific } \\
\text { activity } \\
\left(\mathrm{U} \cdot \mathrm{mg}^{-1}\right. \\
\text { protein })\end{array}$ \\
\hline L0 & none & none & 1.35 & 0.61 & 0.45 & 1.07 \\
\hline L1 & K38G & $\mathrm{K} 38 \mathrm{G}$ & 0.93 & 4.37 & 4.70 & 5.68 \\
\hline L2 & K38G /T40S & K38G /T40S & 1.49 & 8.56 & 5.74 & 12.97 \\
\hline L3 & $\begin{array}{l}\mathrm{S} 36 \mathrm{~A} \\
/ \mathrm{K} 38 \mathrm{G} \\
/ \mathrm{T} 40 \mathrm{~S}\end{array}$ & $\begin{array}{l}\mathrm{S} 36 \mathrm{~A} \\
/ \mathrm{K} 38 \mathrm{G} \\
/ \mathrm{T} 40 \mathrm{~S}\end{array}$ & 0.77 & 4.04 & 5.25 & 5.34 \\
\hline L4 & K151V & K151V & 0.76 & 3.16 & 4.16 & 4.81 \\
\hline L5 & $\begin{array}{l}\text { K151V/ } \\
\text { G167I }\end{array}$ & $\begin{array}{l}\text { K151V/ } \\
\text { G167I }\end{array}$ & 0.68 & 5.76 & 8.47 & 8.35 \\
\hline L6 & $\begin{array}{l}\text { D139V/ } \\
\text { K151V } \\
\text { /G167I }\end{array}$ & $\begin{array}{l}\text { D139V/ } \\
\text { K151V } \\
\text { /G167I }\end{array}$ & 0.62 & 9.18 & 14.81 & 21.03 \\
\hline L7 & $\begin{array}{l}\text { K38G/ } \\
\text { T40S / } \\
\text { D139V/K151V } \\
\text { /G167I }\end{array}$ & $\begin{array}{l}\text { K38G/ } \\
\text { T40S / } \\
\text { D139V/K151V } \\
\text { /G167I }\end{array}$ & 0.59 & 19.45 & 32.97 & 48.94 \\
\hline
\end{tabular}

Figures Legends

Figure 1 Design and reconstruction of main enzyme module and auxiliary module for the synthesis of PAPS. (A) Schematic representation of PAPS biosynthesis from ATP by the main enzymes module (ATPS and APSK) and auxiliary module (PPA and PPK); (B) Effect of different activity ratio of $K l$ ATPS to $P c$ APSK on PAPS production. The dual-enzyme system was supplemented with ATP, with $K l$ ATPS activity fixed at $3.0 \mathrm{U} \cdot \mathrm{mL}^{-1}$. The ratio of $K l$ ATPS to $P c$ APSK was changed from 1:1 to 1:3; (C) Effect of the amount of $E c$ PPA on the conversion rate of PAPS; (D) Effect of the amount of $R s$ PPK on the conversion rate of PAPS.

Figure 2 Construction and optimization of catalytic systemin vivo . (A) Effect of substrate loading on PAPS production by strain E. coli 01 ; The reactions were supplemented with varying concentrations of ATP 
from 20 to $80 \mathrm{mM}$ added at a fixed whole-cell biocatalyst (wet) $30 \mathrm{~g} \cdot \mathrm{L}^{-1}$ at $30^{\circ} \mathrm{C}$; (B) Intracellular enzyme activities of three recombinant strains (E. coli 01,E. coli 04, and E. coli 10); (C) Intracellular enzyme activity of $R c$ PPK in recombinant strains with different chaperones; (D) Intracellular enzyme activity of $P c$ APSK in recombinant strains with different RBS and the effect on the conversion rate of PAPS.

Figure 3 Computer-assisted identification of enzyme structure. (A) The catalytic progress of Pc APSK; (B) Molecular docking and highlighted the predicted hot spots as green sticks; (C) U-shaped release tunnel of ADP and its bottleneck; (D) Root-mean-square fluctuation (RMSF) of Pc APSK; (E) Open and Closed conformation of the lid, and the structure comprises lid constituent residues (red), hinge residues (blue), and binding residues (yellow).

Figure 4 Protein engineering of $P c$ APSK to accelerate ADP release and mechanism analysis. (A) Alanine scanning of selected hot spots; (B) Engineering scheme of Pc APSK; Libraries A and B were constructed using the iterative saturation mutation (ISM) strategies, respectively; Library A had three residues, K38, T40, S36, ISM was used to build the library with an NNK codon; There were three residues, D139, K151, and G167 in library B, which are not close to each other in the structure, and therefore, ISM was used to build the library with an NNK codon. The order is based on the increased enzyme activity (from high to low) of alanine scanning; (C) Hydrogen bonds calculated from MD simulations of L0 and L7; (D) RMSD from MD simulations of L0 and L7; (E) Bottleneck changes in U-shaped tunnel of L0 and L7; (F) Structure alignment of L0 (brown) and L7 (green); (G) Root-mean-square fluctuation (RMSF) value calculated from MD simulations of L0 and L7.

Figure 5 Intracellular enzyme activity and whole cell catalysis. (A) Determination of four intracellular enzyme activities of $E$. coli 11; (B) Conversion experiments of E. coli 04 and E. coli 11 at the 3-L scale with $150 \mathrm{mM}$ ATP. The E. coli04 and E. coli 11 were cultivated in a 5 -L bioreactor (with the 3 -L working volume). Then, the cells were harvested by centrifugation at $6,000 \mathrm{~g}$ for $8 \mathrm{~min}$ and then placed at $35 \operatorname{deg} \mathrm{C}$ for $22 \mathrm{hr}$ for fully self-processing. Moreover, $30 \mathrm{~g}$ cells were resuspended into a 3 - $\mathrm{L}$ of bioconversion mixture (150 Mm ATP, pH was adjusted to 8.0 with $\mathrm{NaOH}$ ). Finally, a total of 3-L volume conversion broth was obtained.

\section{Hosted file}

Figures.pdf available at https://authorea.com/users/408533/articles/518506-rational-designof-a-highly-efficient-catalytic-system-for-the-production-of-3-phosphoadenosine-5phosphosulfate-from-atp 